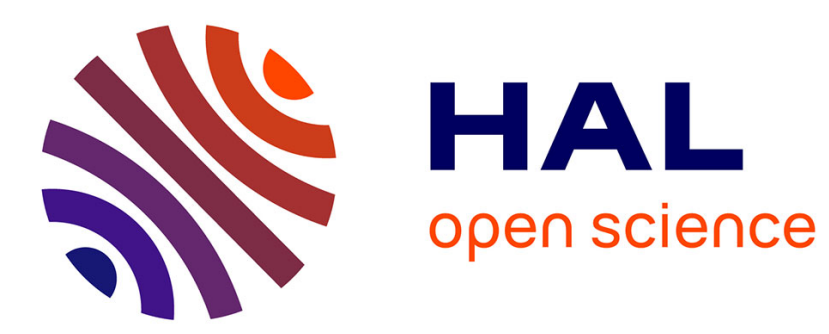

\title{
Seismic response and anisotropy of a model hcp iron inner core
}

\author{
A Lincot, R Deguen, Sébastien Merkel, Philippe Cardin
}

\section{To cite this version:}

A Lincot, R Deguen, Sébastien Merkel, Philippe Cardin. Seismic response and anisotropy of a model hcp iron inner core. Comptes Rendus Géoscience, 2014, 346, pp.148-157. 10.1016/j.crte.2014.04.001 . hal-01260088

\section{HAL Id: hal-01260088 \\ https://hal.science/hal-01260088}

Submitted on 21 Jan 2016

HAL is a multi-disciplinary open access archive for the deposit and dissemination of scientific research documents, whether they are published or not. The documents may come from teaching and research institutions in France or abroad, or from public or private research centers.
L'archive ouverte pluridisciplinaire HAL, est destinée au dépôt et à la diffusion de documents scientifiques de niveau recherche, publiés ou non, émanant des établissements d'enseignement et de recherche français ou étrangers, des laboratoires publics ou privés. 


\title{
Seismic response and anisotropy of a model hcp iron inner core
}

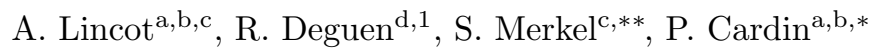 \\ ${ }^{a}$ Univ. Grenoble Alpes, ISTerre, F-38041 Grenoble, France \\ ${ }^{b}$ CNRS, ISTerre, F-38041 Grenoble, France \\ ${ }^{c}$ UMET, CNRS, ENSCL, Université Lille 1, 59655 Villeneuve d'Ascq, France \\ ${ }^{d}$ EPS, Johns Hopkins University, Baltimore, MD 21218, U.S.A
}

\begin{abstract}
We present a framework for simulating the measurement of seismic anisotropy in a model inner core by computing travel time residuals of synthetic seismic rays propagated through the model. The method is first tested on simple inner core structural models consisting of layers with distinct anisotropy, as often proposed in the literature. Those models are not consistent with geodynamics. Hence, we extend the method to a numerically grown inner core composed of $\varepsilon$-Fe with flow generated from an excess of crystallization in the equatorial belt, inducing polycrystalline textures. The global inner core anisotropy is 7 times smaller than that of the single crystal. Compositional stratification amplifies the global anisotropy by $15 \%$ while the addition of solidification textures reduces it by a factor of 2 . As such, and within the tested geodynamical models, no published elastic model of $\varepsilon$-Fe at inner core conditions allows for reproducing the $3 \%$ cylindrical anisotropy reported in seismology publications. In addition, our models demonstrate that additional information such as the depth dependence and the spread of the observed anisotropy is a key for revealing the dynamics and history of the inner core.
\end{abstract}

Keywords: inner core, anisotropy, iron, texture, geodynamical model, seismic wave

\section{Introduction}

Compressional seismic body waves differential travel time residuals and free oscillations reveal a large scale cylindrical anisotropy in the Earth's inner core: seismic waves travel faster along the north-south (NS) axis than within the equatorial plane (Poupinet et al., 1983; Morelli et al., 1986;

5 Woodhouse et al., 1986). Over the following years, this observation was refined with evidences of both hemispherical and radial variations of the anisotropy level and geometry. Recent studies indi-

\footnotetext{
*Principal corresponding author

${ }^{* *}$ Corresponding author

Email addresses: sebastien.merkel@univ-lille1.fr (S. Merkel), philippe.cardin@ujf-grenoble.fr (P. Cardin)

${ }^{1}$ Present address: Laboratoire de Géologie de Lyon, UMR5276, Université Claude Bernard Lyon 1 - CNRS ENS Lyon, Villeurbanne, France.
} 
cate up to $4.4 \%$ and $1 \%$ anisotropy in the western and eastern hemispheres, respectively (Tanaka and Hamaguchi, 1997; Garcia and Souriau, 2000; Niu and Chen, 2008; Deuss et al., 2010; Irving and Deuss, 2011), with an almost isotropic layer - $60 \mathrm{~km}$ deep in the most recent studies - at the top of the inner core surrounding a more anisotropic region (Shearer, 1994; Song and Helmberger, 1995; Creager, 1999; Garcia and Souriau, 2000; Waszek and Deuss, 2011), and, possibly, an innermost inner core with yet different properties (Ishii and Dziewoński, 2002; Beghein and Trampert, 2003; Calvet et al., 2006; Niu and Chen, 2008; Sun and Song, 2008b,a), although the presence of an innermost inner core is not required in the analysis of more recent datasets (Lythgoe et al., 2013). In this paper, we present models aiming at reproducing global NS anisotropy and its depth variation.

Seismic anisotropy observed at the global scale is the signature of single crystal anisotropy of the solid material in the inner core. For simplicity, we consider an inner core made of pure iron as other "light" elements are not clearly identified (e.g. Alfè et al., 2002; Badro et al., 2007; Antonangeli et al., 2010; Tsuno et al., 2013). Although other phases have been proposed (Vočadlo et al., 2003; Dubrovinsky et al., 2007; Kuwayama et al., 2008; Belonoshko et al., 2008), we consider a pure $h c p$ - or $\varepsilon$-Fe inner core, in agreement with recent experimental studies (Tateno et al., 2010), and assume dominant basal slip, with contributions of prismatic and pyramidal slip (Merkel et al., 2012).

Global anisotropy requires the alignment of anisotropic single crystals. Processes for generating such textures include solidification texturing acquired at the inner core boundary (ICB) during the growth of the inner core (Bergman, 1997; Brito et al., 2002) and/or plastic deformation due to internal dynamics after the solidification (Jeanloz and Wenk, 1988; Yoshida et al., 1996; Karato, 1999; Deguen and Cardin, 2009; Takehiro, 2010). Those models often results in complex textures that can be difficult to interpret at the global scale. As such, the interpretation of seismic data is often simplified by introducing "structural" inner core models, with different layers of distinct anisotropy (e.g. Stixrude and Cohen, 1995; Bergman, 1997; Calvet et al., 2006; Sun and Song, 2008a; Lythgoe et al., 2013).

In this paper, we address the issue of scaling microscopic properties such as single-crystal elasticity of $\varepsilon$-Fe to the macroscopic observations of seismic travel times. We first study the seismic response and estimate the anisotropy of structural model inner cores, based on procedures used in seismological studies. We later extend the results by coupling a dynamical model of inner core formation with a numerical model of texture development in $\varepsilon$-Fe, computing the time evolution of textures in the inner core, and simulating the measurement of seismic anisotropy. At last, we discuss the synthetic global anisotropy in comparison to the observed results in order to deduce conclusions for the inner core. 


\section{Structural models}

\subsection{Inner core structural models}

Following procedures used in previous studies (Stixrude and Cohen, 1995; Bergman, 1997;

45 Calvet et al., 2006; Sun and Song, 2008a; Lythgoe et al., 2013), we first build simple structural inner core models. In each model, the inner core is built with different layers with distinct anisotropies (Figs. 1, 2).

Elastic properties of $\varepsilon$-Fe at inner core conditions from first principles models and experiments are still debated (e.g. Stixrude and Cohen, 1995; Mao et al., 1998; Laio et al., 2000; Steinle-Neumann et al., 2001; Antonangeli et al., 2006; Vočadlo et al., 2009; Sha and Cohen, 2010; Martorell et al., 2013) and do not especially match those of the inner core. Here, we intend to understand the seismic response of simple models, extracting the signature of the inner core substructure on the depth and orientation dependence of travel time residuals. As such, and although they will affect the numerical values of global seismic anisotropy, the actual values of elastic moduli we use are not the main focus in our analysis.

Seismic waves are faster along the NS direction than in the equatorial plane. Considering anisotropy at the scale of a single crystal, this matches the symmetry of wave propagation in an $h c p$ single crystal with the $c$-axis faster than the $a$-axis. We therefore choose a set of elastic constants for $\varepsilon$-Fe with the $c$-axis faster than the $a$-axis. Among published sets of elastic moduli

60 for $\varepsilon$-Fe, we select the results of Laio et al. (2000) that agree with this constraint with single crystal elastic moduli (in GPa) $\mathrm{C}_{11}=1554 ; \mathrm{C}_{12}=742 ; \mathrm{C}_{13}=820 ; \mathrm{C}_{33}=1796 ; \mathrm{C}_{44}=414$. We then build two polycrystals with various levels of alignment of the $c$-axes of the crystallites $(100 \%$, $50 \%$, Figs. 1a, b), producing two materials with distinct anisotropic signatures, later labeled as "strongly" and "mildly" anisotropic. Solidification textures have also been proposed as a source for anisotropy in the inner core (Bergman, 1997; Brito et al., 2002). For hcp materials, solidification textures align the $c$-axes of the polycrystal perpendicular to the solidification front (Bergman, 1997; Brito et al., 2002; Bergman et al., 2003). Fig. 1c presents the anisotropy of such a polycrystal. This model will be labeled as "dendritic".

Considering the diversity of inner core anisotropy models published in the literature, we inves70 tigate the response of eight different structural models (Fig. 2):

a) an homogeneous inner core with mildly anisotropic elastic properties aligned with the Earth rotation axis,

b) an homogeneous inner core with mildly anisotropic elastic properties tilted $10^{\circ}$ away from the axis of rotation,

75 c) a mildly anisotropic inner core surrounded by a $150 \mathrm{~km}$ deep isotropic region, 
d) an inner core with a mildly anisotropic outer inner core and a dentritic inner inner core of $550 \mathrm{~km}$ radius,

e) an inner core with a mildly anisotropic outer inner core and an isotropic inner inner core of $550 \mathrm{~km}$ radius,

so f) an inner core with mildly anisotropic outer inner core and a strongly anisotropic inner inner core of $550 \mathrm{~km}$ radius,

g) an inner core with a spherical crystallization and dentritic elastic properties,

h) an inner core with a cylindrical crystallization and dendritic elastic properties.

More than 100000 synthetic seismic rays are randomly generated to probe the whole inner core and simulate seismic measurements (Fig. 3). For each ray, we then estimate the normalized seismic travel time residual

$$
\delta t / t=\frac{s^{0}-s}{s^{0}}
$$

where $s$ is the simulated slowness of the seismic ray and $s^{0}$ is the slowness of that same ray for an homogeneous and fully isotropic inner core.

\subsection{Results for single-layer inner cores}

In our first structural model, with a mildly anisotropic inner core aligned with the rotation axis (Fig. 2a), the inner core behaves as a giant single crystal with its $c$ axis aligned with the NS axis and a minimum velocity in the equatorial plane. The global anisotropy is equal to the anisotropy at the microscopic scale and travel time residuals are independent of the depth of the ray turning point. Provided enough anisotropy at the single crystal scale, it can match the observed data in terms of global anisotropy.

The second structural model (Fig. 2b) consists of a mildly anisotropic inner core, with the fast axis tilted by $10^{\circ}$ from the Earth's rotation axis, as suggested by some seismic models (Su and Dziewonski, 1995; Romanowicz et al., 1996; Song and Richards, 1996). The $10^{\circ}$ tilt produces a spread of travel time residuals that, in average, look similar to those of the model in Fig. 2a. As in the previous case, there is no depth dependence of the travel time residuals.

\subsection{Effect of an isotropic superficial layer}

Other seismological models (Shearer, 1994; Song and Helmberger, 1995; Creager, 1999; Garcia and Souriau, 2000; Waszek and Deuss, 2011) lead to an isotropic layer at the top of the inner core. Such models, with a $150 \mathrm{~km}$ thick isotropic region (Fig. 2c), produce no anisotropy for superficial rays while the deeper rays, below $\approx 250 \mathrm{~km}$, follow a similar trend as observed for an inner core with homogeneous properties, with a reduced amplitude of the anisotropy relative to that of the 
polycrystal model, and with mean anisotropy increasing with the depth of the turning point. With an isotropic layer reduced to $60 \mathrm{~km}$, the transition to anisotropy will be more superficial while deeper rays will show little difference to those obtained for a fully homogeneous inner core. Also note that, for a given depth of turning point, there is no spread of travel time residuals.

\subsection{Effect of an innermost inner core}

Ishii and Dziewoński (2002) proposed an innermost inner core of $300 \mathrm{~km}$ radius that exhibits distinct elastic properties relative to the bulk inner core. This is supported by more recent studies

115 (e.g. Calvet et al., 2006; Beghein and Trampert, 2003; Niu and Chen, 2008; Sun and Song, 2008b,a), at perhaps a larger radius of 400-550 km. This hypothesis is tested in Figs. 2d, e, and $\mathrm{f}$ assuming an inner inner core radius of $550 \mathrm{~km}$. $550 \mathrm{~km}$ lies in the upper limit of innermost inner core radius estimates (Calvet et al., 2006; Sun and Song, 2008a). However, we intend here to investigate whether such innermost inner core can be detected using differential travel times and its effect of global anisotropy. As such, our conclusion are not heavily dependent on the exact radius of this innermost inner core.

Our first model (Fig. 2d) consists of a mildly anisotropic outer inner core with an inner inner core with opposite elastic properties, i.e., with the slow axes of the polycrystal aligned with the Earth rotation axis. Travel time anomalies are similar to those of Fig. 2a for superficial rays. Anisotropy decreases with depth with, therefore, a strong depth dependence of the residuals.

Results are similar if the inner inner core is fully isotropic (Fig. 2e), with travel time residuals similar to those of Fig. 2a for superficial rays and a decrease of anisotropy with depth.

An inner inner core with strongly anisotropic properties (Fig. 2f) with similar properties than the outer inner core, hence twice stronger, produces similar measurements that in Fig. 2a for superficial rays, with an increase of anisotropy with depth. Do note, however, that the increase of anisotropy with depth is small and could be difficult to detect in actual seismic measurements.

\subsection{Dendritic models}

Finally, solidification texturing during the formation of the inner core was also suggested as a possible source of inner core anisotropy (Bergman, 1997; Brito et al., 2002). In this hypothesis, inner core anisotropy is a record of the formation of the inner core.

Our first model (Fig. 2g) considers a radial dendritic growth of the inner core (Fearn et al., 1981). In this model, the slow directions of the polycrystal align in a radial direction, resulting in an inner core with spherical elastic symmetry. Travel time residuals depend on the depth of turning point of the ray. They are insensitive to the angle to the NS axis. This model is, hence, isotropic seismically. 
An alternative dendritic model is based on a cylindrical growth (Fig. 2h) of the inner core (Bergman, 1997). In this model, the direction of solidification is perpendicular to the Earth rotation axis. $c$-axes of the polycrystal are perpendicular to the solidification front and, hence, align on a cylindrical surface parallel to the Earth rotation axis. In this case, $a$-axes in the polycrystal

\subsection{Overview of inner core geodynamical models}

The core crystallizes from the center outward because the melting temperature of the core mixture increases with depth faster than the (adiabatic) core geotherm. Inner core crystallization 
induces potential deformation and, hence, development of anisotropy inside the inner core. Such processes, coupled with radiogenic heating, have been proposed to induce a thermal forcing as a drive for convection (Jeanloz and Wenk, 1988; Buffett, 2009), with a potential chemical stratification (Deguen and Cardin, 2011). Other models for generating deformation inside the core include magnetic forcing (Karato, 1999; Buffett and Wenk, 2001; Takehiro, 2010) or heterogeneous growth of the inner core controlled by thermal motions in the outer core (Yoshida et al., 1996; Deguen et al., 2011).

Most of those models are axisymmetric and, hence, can not account for the hemispheric dependence of seismological data. To address this issue, geodynamical models considering a thermal translation of the core were recently considered in the literature (Monnereau et al., 2010; Alboussière et al., 2010; Deguen et al., 2013; Mizzon and Monnereau, 2013).

Our study focuses on differential growth mechanisms (Fig. 4), assuming that geostrophic convection in the outer core results in faster crystallization in the equatorial belt of the inner core. The resulting topography is continuously relaxed by a quadrupolar viscous flow within the inner core (Yoshida et al., 1996), named hereafter Yos-Ran. The reason for this choice is that, among published models, this model generates a large amount of large scale deformation in the inner core and that, thanks to its simplicity, it is a good test case for investigating the development of anisotropy with deformation. Anisotropy in such model can be enhanced by the addition of solidification induced pre-texturation at the ICB, named Yos-Ptex, and deformation due to the bulk effect of a stable density stratification which localizes the motions by minimizing any radial motion, named Yos-Strat. Non axisymmetric models, such as those involving thermal translation of the inner core, are out of the scope of this study, which focuses on models preserving a symmetry around the axis of rotation of the Earth.

\section{2. $\varepsilon$-Fe texture induced by the geodynamical model}

Our model is based on the code described in Deguen and Cardin (2011), with neutral chemical stratification $(B=0)$ and an equatorial growth rate twice faster than at the poles $\left(S_{2}=2 / 5\right)$. Continuous relaxation of the dynamic topography results in a flow from the equator to the poles with a quadrupolar pattern inside the whole inner core. Using methods developed in a previous study (Deguen et al., 2011), we compute the position and deformation for 100 markers introduced at the ICB during inner core growth. Texture along markers are then calculated for a 3000 grains aggregate of $\varepsilon$-Fe using the Los Alamos viscoplastic self-consistent (VPSC) code of Lebensohn and Tomé (1993).

In the Yos-Ran model, the aggregate is created at the ICB with a random texture, i.e. with an equal probability for any crystal orientations. We assume dominant basal slip, with contributions 
of prismatic and pyramidal slip (Merkel et al., 2012). In this model, texture is weak in the outer portions of the inner core and only significant closer to the center of the inner core. It correspond polar axis of the inner core (Fig. 5a).

Model Yos-Strat includes the effect of a chemical or thermal stratification within the inner core $\left(B^{*}=-10^{6}\right.$ in the model of Deguen et al., 2011). Stratification suppresses radial motion in the bulk of the inner core. The equatorial bulge is relaxed by plastic flow within a superficial of the inner core while, near the center, textures are weak due to the small deformation applied to the polycrystal (Fig. 5b).

Textures are weak in the outer regions of model Yos-Ran. In parallel, multiple studies suggested solidification textures as a potential source of inner core anisotropy (Bergman, 1997; Brito et al.,

In order to obtain seismic residuals with a fast NS axis, compression wave velocities of $\varepsilon$-Fe in such model should be maximum along the $a$-axis. This requirement is the opposite of that of structural models described in the previous section. As such, the set of elastic moduli selected previously (Laio et al., 2000) will not allow for a successfully model inner core anisotropy.

Recent published models (Steinle-Neumann et al., 2001; Vočadlo et al., 2009; Sha and Cohen, 2010) exhibit such velocity profiles for $\varepsilon$-Fe at core conditions. In the results of Sha and Cohen (2010), the anisotropy of $\varepsilon$-Fe single crystals at core condition lies in the order of $1 \%$. This quasi elastic isotropy is problematic for explaining inner core anisotropy. The results of Steinle-Neumann et al. (2001) do provide large anisotropy at core conditions but the approximation used in those calculations has been controversial (Gannarelli et al., 2003). Consequently, we select the published results of Vočadlo et al. (2009) at $5500 \mathrm{~K}$ with single crystal elastic moduli (in GPa) $\mathrm{C}_{11}=1646$; $\mathrm{C}_{12}=1253 ; \mathrm{C}_{13}=995 ; \mathrm{C}_{33}=1559 ; \mathrm{C}_{44}=153$. These numbers imply a sound velocity along the $c$-axis $5 \%$ slower than that along the $a$-axis.

At each point of the grid of each geodynamical model (Fig. 5), the local elastic tensor is calculated by weighting the single crystal elastic tensor with the aggregate texture, in the Hill approximation. Solving the Christoffel equations, we are then able to calculate $P$-wave velocities 
for any direction of propagation (Fig. 6). Travel time residuals are then calculated using the procedure described in Figure 3.

Our model allows for a detailed analysis of anisotropy, including the depth and orientation dependence of the travel time residuals (Fig. 7). However, seismological studies usually do not have the resolution to investigate the full details of inner core anisotropy. Different strategy were put forward to overcome the small amount of data. A global view could be obtained by using spherical harmonics (Poupinet et al., 1983). Alternatively, it was proposed to fit travel times residuals using (Creager, 1992)

$$
\delta t / t=a+b \cos ^{2} \zeta+c \cos ^{4} \zeta
$$

where $a, b$ and $c$ are adjustable parameters and $\zeta$ is the angle between the ray and the Earth rotation axis. The quantity $b+c$ is the difference between polar $\left(\zeta=0^{\circ}\right)$ and equatorial $\left(\zeta=90^{\circ}\right)$ residuals, and is a measure of the global inner core anisotropy often reported in the literature. Recent results by Irving and Deuss (2011) on their entire catalog of $\mathrm{PKP}_{d f-b c}$ differential travel time residuals reveal a 3.3 to $3.8 \%$ global NS anisotropy. This observation is regionally dependent:

255 a subset of data in the western hemisphere leads to an anisotropy ranging from 4.5 to $4.8 \%$ while the eastern hemisphere is only 0.5 to $1.4 \%$ anisotropic.

\subsection{Results for model Yos-Ran}

For this model, we find positive time residuals for rays parallel to the axis of rotation $\left(\right.$ at $0^{\circ}$, Fig. 7a). Equatorial path travel time residuals are small, sometimes negative: seismic waves travel faster along polar paths. The global shape of the time residuals is similar to seismic observations, even if actual polar observations are scarce.

Travel time residuals do not exceed 1\%. As such, this model can not account for global seismological observations. Reasons from this disagreement are as follow. i) Aggregates with the strongest textures, in the inner portions of the inner core do not display such simple anisotropy

features as a pure single crystal of $\varepsilon$-Fe and, hence, are averaged out over the path of the ray. ii) In this model, most of the inner core is isotropic and hence, contributes to an isotropic signal at the global scale.

This model, on the other hand, clearly displays a depth dependence of the time residuals: rays with deeper turning points would lead to a larger anisotropy. This is in broad agreement with seismic observations. In this case, deformation in the superficial layers of the model is small. Consequently, the outer portion of the core is nearly isotropic and would lead to negligible travel time residuals, whatever the direction of propagation of the ray.

The fit of Eq. 2 to the data leads to a global anisotropy of 0.7\% (Figs. 7a). This global anisotropy is clearly below most seismological estimates. Nevertheless, the shape of the anisotropy as a 
flow, the single crystal anisotropy should increase by a factor of $\sim 5$ in order to match the reported global anisotropy. In other words, with such model, a single crystal anisotropy on the order of $15 \%$ would be required for a $3 \%$ global seismic anisotropy.

\subsection{Results for model Yos-Strat}

Chemical stratification concentrates textures in upper portions of the inner core (Fig. 5b). In this model, the anisotropy is dominated by fast $P$-wave velocities in the horizontal direction (Fig. 6b). At intermediate depths, we can observe some component of NS anisotropy, reminiscent of the features of the non-stratified model.

The travel time residuals are complex and spread over almost 3\% (Fig. 7b). As expected, the On the other hand, the fit of Eq. 2 to the data leads to a global anisotropy of $0.8 \%$ (Figs. $7 \mathrm{~b}$ ). In average, this model does lead to faster waves along the NS axis than in the equatorial plane, as observed in seismology. Data points, on the other hand, are spread and complex, with residuals found far away from the fit of Eq. 2.

The depth dependence of the anisotropy is opposite to seismic observations: anisotropy is low for the deepest rays while travel times for shallower rays do set a trend for a global NS anisotropy.

This model does not broadly agree with seismic observations. On the other hand, and as already shown with the structural models, these results emphasize how the spread and depth dependence of travel time residuals hold important information. Both are strongly affected by structural features

\subsection{Results for model Yos-Ptex}

Relative to model Yos-Ran, ICB crystallization textures in model Yos-Ptex enhances anisotropy in the outer regions of the inner core (Fig. 6c). In the deeper portions of the inner core, crystallization textures are largely erased by plastic deformation and the resulting velocities resemble that of model Yos-Ran. Due to the spherical symmetry of the crystallization process, $P$-wave velocity profiles in the shallower portions of the inner core do display a strong spherical symmetry.

Travel time residuals lie between $-0.05 \%$ and $2 \%$ (Fig. $7 \mathrm{c}$ ). Also note the very strong depth dependence of the residual. On the other hand, the average global anisotropy of such model is low, of the order of $0.3 \%$, due to the spherical symmetry of the crystallization textures. Whatever the with faster arrivals for deeper rays. As such, this model is not in line with seismic observations. 


\section{Discussion and conclusion}

We developed a new method for scaling microscopic properties such as single-crystal elasticity of $\varepsilon$-Fe to the macroscopic observations of seismic travel times. Using elastic models for $\varepsilon$-Fe published in the literature, it appears difficult to obtain a 3.3 to $3.8 \%$ global anisotropy, as proposed in recent works (Irving and Deuss, 2011). Calculations were repeated for other published models for $\varepsilon$-Fe (Stixrude and Cohen, 1995; Söderlind et al., 1996; Laio et al., 2000; Steinle-Neumann et al., 2001; Sha and Cohen, 2010) and, within the scope of our geodynamical models, the single crystal anisotropy was not sufficient to match that observed in inner core seismic studies.

Elastic moduli of Steinle-Neumann et al. (2001) do allow for a $2 \%$ global anisotropy whereas, in recent years, calculations have converged towards more isotropic models (e.g. Vočadlo et al., 2009; Sha and Cohen, 2010). It should be noted, however, that dynamics simulations suggest a strong non-linear behavior of $\varepsilon$-Fe at $360 \mathrm{GPa}$, just before melting (Martorell et al., 2013). This strongly nonlinear effect in Fe could occur in the inner core and, hence, provide enough anisotropy to match seismic observations. Anisotropy at the single crystal scale may also be increased by including the effect of elements other than Fe.

Using simple structural models and a proper set of elastic moduli, it is possible to match seismic observations. We find no measurable anisotropy for rays propagating at small depth when a superficial isotropic layer is considered. The existence of an innermost inner core may be unraveled 25 by a depth dependence of the residuals but it is difficult to strictly identify the elasticity of the material in the central region. We also note that it is not straightforward to conclude on the sharpness of internal interfaces between regions of different elasticity with travel time data because of the integration of the residuals over the whole inner core portion of the ray.

Structural models provide an easy interpretation of the seismic data. On the other hand, those models offer little clues regarding the dynamics and the physical processes at play during the formation of the inner core. In more realistic models such as random and fast crystallization at the equator (Yoshida et al., 1996, i.e. Yos-Ran), averaging along seismic rays reduces the global anisotropy by a factor of 7 relative to that of the single crystal. A strong local anisotropy is reduced at the global scale by other regions where the anisotropy displays a different orientation. Stratification produces a complex and localized deformation and anisotropy, stronger than in YosRan. Despite averaging effects, the global anisotropy increases by about 15\%, with an apparent symmetry around the Earth rotation axis. Solidification textures, on the other hand, introduce a spherical symmetry in the local anisotropy. Global anisotropy is then reduced by a factor of 2 .

We observe a strong depth dependence of the PKP wave travel time residuals that depends on the model. Spreading of data points is also a key for differentiating the different geodynamical 
models. As such, we find that simple models such as Yos-Ran are more in line with seismic observations relative to models including stratification or solidification textures for which the depth dependence and spread of travel time residuals is wrong. This emphasizes that a simple fit as in Eq. 2 through the dataset oversimplifies the data. Such fit smooths out the depth dependence and the spread of the travel time residuals which are deeply related to the tectonics of the inner core.

Finally, it is likely that the scarce geographical coverage of seismic data does not correctly probe the full inner core. As such, it would be of interest to characterize the under-sampling of actual observations. Numerical models, such as those presented here, could be used to investigate the reliability of the observational constraints using actual entry and exit points of ray paths used in seismic studies.

We therefore suggest that the analysis and parametrization of seismic data progress towards constraining the depth and geographical dependence of travel time residuals, with additional information on the spread, to allow for a better characterization of processes of inner core formation.

\section{Acknowledgments}

The authors wish to thank the reviewers and the Geodynamo group for useful discussions. This work has been financed by the program PNP of CNRS/INSU, OSUG@2020, and the doctorate program of Université Joseph Fourier. Calculations have been made at Centre de Calcul Commun of OSUG. RD was supported by grant EAR-0909622 from the National Science Foundation.

\section{References}

Alboussière, T., Deguen, R., Melzani, M., 2010. Melting induced stratification above the Earth's inner core due to convective translation. Nature 466, 744-747.

Alfè, D., Price, G.D., Gillan, M.J., 2002. Iron under Earth's core conditions: Liquid-state thermodynamics and high-pressure melting curve from ab initio calculations. Phys. Rev. B 65, 165118.

Antonangeli, D., Merkel, S., Farber, D.L., 2006. Elastic anisotropy in hcp metals at high pressure and the sound wave anisotropy of the Earth's inner core. Geophys. Res. Lett. 33, L24303.

Antonangeli, D., Siebert, J., Badro, J., Farber, D.L., Fiquet, G., Morard, G., Ryerson, F.J., 2010. Composition of the Earth's inner core from high-pressure sound velocity measurements in FeNi-Si alloys. Earth Planet. Sci. Lett. 295, 292-296. doi:10.1016/j.epsl.2010.04.018. 
Badro, J., Fiquet, G., Guyot, F., Gregoryanz, E., Occelli, F., Antonangeli, D., D’Astuto, M., 2007. Effect of light elements on the sound velocities in solid iron: Implications for the composition of Earth's core. Earth Planet. Sci. Lett. 254, 233-238.

Beghein, C., Trampert, J., 2003. Robust normal mode constraints on inner-core anisotropy from model space search. Science 299, 552-555.

Belonoshko, A.B., Skorodumova, N.V., Rosengren, A., Johansson, B., 2008. Elastic anisotropy of Earth's inner core. Science 319, 797-800.

Bergman, M.I., 1997. Measurements of elastic anisotropy due to solidification texturing and the implications for the Earth's inner core. Nature 389, 60-63.

Bergman, M.I., Agrawal, S., Carter, M., Macleod-Silberstein, M., 2003. Transverse solidification textures in hexagonal close-packed alloys. J. Crystal Growth 255, 204-211.

Brito, D., Elbert, D., Olson, P., 2002. Experimental crystallization of gallium: ultrasonic measurements of elastic anisotropy and implications for the inner core. Phys. Earth Planet. Inter. 129, 325-346. doi:10.1016/S0031-9201(01)00298-9.

Buffett, B.A., 2009. Onset and orientation of convection in the inner core. Geophys. J. Int. 179, 711-719.

Buffett, B.A., Wenk, H.R., 2001. Texturing of the Earth's inner core by Maxwell stresses. Nature 413, 60-63.

Calvet, M., Chevrot, S., Souriau, A., 2006. P-wave propagation in transversely isotropic media: II. Application to inner core anisotropy: Effects of data averaging, parametrization and a priori information. Phys. Earth Planet. Inter. 156, 21-40.

Creager, K.C., 1992. Anisotropy of the inner core from differential travel times of the phases PKP and PKIKP. Nature 356, 309-314.

Creager, K.C., 1999. Large-scale variations in inner core anisotropy. J. Geophys. Res. 104, 2312723139.

Deguen, R., Alboussiere, T., Cardin, P., 2013. Thermal convection in Earth's inner core with phase change at its boundary. Geophys. J. Intern. 194, 1310-1334. doi:10.1093/gji/ggt202.

Deguen, R., Cardin, P., 2009. Tectonic history of the Earth's inner core preserved in its seismic structure. Nature. Geosci. 2, 419-422. 
Deguen, R., Cardin, P., 2011. Thermo-chemical convection in Earth's inner core. Geophys. J. Int. 187, 1101-1118.

Deguen, R., Cardin, P., Merkel, S., Lebensohn, R.A., 2011. Texturing in Earth's inner core due to preferential growth in its equatorial belt. Phys. Earth Planet. Inter. 188, 173-184. doi:10.1016/j.pepi.2011.08.008.

Deuss, A., Irving, J., Woodhouse, J., 2010. Regional variation of inner core anisotropy from seismic normal mode observations. Science 328, 1018-1020.

Dubrovinsky, L., Dubrovinskaia, N., Narygina, O., Kantor, I., Kuznetzov, A., Prakapenka, V.B., Vitos, L., Johansson, B., Mikhaylushkin, A.S., Simak, S.I., Abrikosov, I.A., 2007. Body-centered cubic iron-nickel alloy in Earth's core. Science 316, 1880-1883.

Fearn, D., Loper, D., Roberts, P., 1981. Structure of the Earth's inner core. Nature 292, 232-233.

Gannarelli, C.M.S., Alfè, D., Gillan, M.J., 2003. The particle-in-cell model for ab initio thermodynamics: implications for the elastic anisotropy of the Earth's inner core. Phys. Earth Planet. Inter. 139, 243-253.

Garcia, R., Souriau, A., 2000. Inner core anisotropy and heterogeneity level. Geophys. Res. Lett. $27,3121-3124$.

Irving, J.C.E., Deuss, A., 2011. Hemispherical structure in inner core velocity anisotropy. J. Geophys. Res. 116, B04307. doi:10.1029/2010JB007942.

Ishii, M., Dziewoński, A.M., 2002. The innermost inner core of the Earth: Evidence for a change in anisotropic behavior at the radius of about $300 \mathrm{~km}$. Proc. Natl. Acad. Sci. 99, 14026-14030.

Jeanloz, R., Wenk, H., 1988. Convection and anisotropy of the inner core. Geophys. Res. Lett. 15, 420 $72-75$.

Jeanloz, R., Wenk, H.R., 1988. Convection and anisotropy of the inner core. Geophys. Res. Lett. $15,72-75$.

Karato, S., 1999. Seismic anisotropy of the Earth's inner core resulting from flow induced by Maxwell stresses. Nature 402, 871-873.

${ }_{425}$ Kuwayama, Y., Hirose, K., Sata, N., Ohishi, Y., 2008. Phase relations of iron and iron-nickel alloys up to $300 \mathrm{GPa}$ : Implications for composition and structure of the earth's inner core. Earth Planet. Sci. Lett. 273, 379-385. 
Laio, A., Bernard, S., Chiarotti, G.L., Scandolo, S., Tosatti, E., 2000. Physics of iron at Earth's core conditions. Science 287, 1027-1030.

Lebensohn, R.A., Tomé, C.N., 1993. Self-consistent anisotropic approach for the simulation of plastic deformation and texture development of polycrystals: application to zirconium alloys. Acta Metall. Mater. 41, 2611-2624.

Lythgoe, K., Deuss, A., Rudge, J., Neufeld, J., 2013. Earths inner core: Innermost inner core or hemispherical variations? Earth Planet. Sci. Lett. 385, 181-189. doi:10.1016/j.epsl.2013.10.049.

Mao, H.K., Shu, J., Shen, G., Hemley, R.J., Li, B., Singh, A.K., 1998. Elasticity and rheology of iron above 220GPa and the nature of the Earth's inner core. Nature 396, 741-743.

Martorell, B., Vočadlo, L., Brodholt, J., Wood, I.G., 2013. Strong premelting effect in the elastic properties of hcp-fe under inner-core conditions. Science 342, 466-468. doi:10.1126/science.1243651.

Merkel, S., Gruson, M., Wang, Y., Nishiyama, N., Tomé, C.N., 2012. Texture and elastic strains in hcp-iron plastically deformed up to $17.5 \mathrm{GPa}$ and $600 \mathrm{~K}$ : experiment and model. Modelling Simul. Mater. Sci. Eng. 20, 024005. doi:10.1088/0965-0393/20/2/024005.

Mizzon, H., Monnereau, M., 2013. Implication of the lopsided growth for the viscosity of earth's inner core. Earth Planet. Sci. Lett. 361, 391-401.

Monnereau, M., Calvet, M., Margerin, L., Souriau, A., 2010. Lopsided growth of Earth's inner core. Science 328, 1014-1017.

Morelli, A., Dziewonski, A.M., Woodhouse, J.H., 1986. Anisotropy of the inner core inferred from PKIKP travel times. Geophys. Res. Lett. 13, 1545-1548.

Niu, F., Chen, Q., 2008. Seismic evidence for distinct anisotropy in the innermost inner core. Nat. Geosci. 1, 692-696. doi:10.1038/ngeo314.

Poupinet, G., Pillet, R., Souriau, A., 1983. possible heteregeneity of the Earth's core deduced from PKIKP travel-times. Nature 305, 204-206.

Romanowicz, B., Li, X., Durek, J., 1996. Anisotropy in the inner core: Could it be due to low-order convection? Science 274, 963-966. doi:10.1126/science.274.5289.963.

Sha, X., Cohen, R.E., 2010. Elastic isotropy of $\epsilon$-Fe under Earth's core conditions. Geophys. Res. Lett. 37, L10302. doi:10.1029/2009GL042224. 
Shearer, P.M., 1994. Constraints on inner core anisotropy from PKP(DF) travel times. J. Geophys. Res. 99, 19647-19659. URL: http://dx.doi.org/10.1029/94JB01470, doi:10.1029/94JB01470.

Söderlind, P., Moriarty, J.A., Wills, J.M., 1996. First-principles theory of iron up to earth-core pressures: Structural, vibrational, and elastic properties. Phys. Rev. B 53, 14063-14072. URL: http://link.aps.org/doi/10.1103/PhysRevB.53.14063, doi:10.1103/PhysRevB.53.14063.

Song, X., Helmberger, D.V., 1995. Depth dependence of anisotropy of Earth's inner core. J. Geophys. Res. 100, 9805-9816. doi:10.1029/95JB00244.

Song, X., Richards, P., 1996. Seismological evidence for differential rotation of the earth's inner core. Nature 382, 221-224. doi:10.1038/382221a0.

Souriau, A., 2003. The seismological picture of the inner core: structure and rotation. C. R. Geosci. 335, 51-63. doi:10.1016/S1631-0713(03)00010-5.

Steinle-Neumann, G., Stixrude, L., Cohen, R.E., Gülseren, O., 2001. Elasticity of iron at the temperature of the Earth's inner core. Nature 413, 57-60.

Stixrude, L., Cohen, R.E., 1995. High-pressure elasticity of iron and anisotropy of Earth's inner core. Science 267, 1972-1975.

Stixrude, L., Cohen, R.E., 1995. High-pressure elasticity of iron and anisotropy of Earth's inner core. Science 267, 1972-1975.

Su, W., Dziewonski, A.M., 1995. Inner core anisotropy in three dimensions. J. Geophys. Res. 100, 9831-9852.

Sun, X., Song, X., 2008a. The inner inner core of the Earth: Texturing of iron crystals from three-dimensional seismic anisotropy. Earth Planet. Sci. Lett. 269, 56-65.

Sun, X., Song, X., 2008b. Tomographic inversion for three-dimensional anisotropy of earths inner core. Phys. Earth Planet. Inter. 167, 53-70.

Takehiro, S., 2010. Fluid motions induced by horizontally heterogeneous joule heating in the Earth's inner core. Phys. Earth Planet. Inter. 184, 134-142.

Tanaka, S., Hamaguchi, H., 1997. Degree one heterogeneity and hemispherical variation of anisotropy in the inner core from PKP(BC)-PKP(DF) times. J. Geophys. Res. 102, 2925-2938. doi:10.1029/96JB03187.

${ }_{485}$ Tateno, S., Hirose, K., Ohishi, Y., Tatsumi, Y., 2010. The structure of iron in Earth's inner core. Science 330, 359-361. 
Tsuno, K., Frost, D.J., Rubie, D.C., 2013. Simultaneous partitioning of silicon and oxygen into the Earth's core during early earth differentiation. Geophys. Res. Lett. 40, 66-71. doi:10.1029/2012GL054116.

Vočadlo, L., Alfè, D., Gillan, M.J., Wood, I., Brodholt, J., Price, G.D., 2003. Possible thermal and chemical stabilization of body-centred-cubic iron in the Earth's core. Nature 424, 536-539.

Vočadlo, L., Dobson, D.P., Wood, I.G., 2009. Ab initio calculations of the elasticity of hcp-Fe as a function of temperature at inner-core pressure. Earth Planet. Sci. Lett. 288, 534-538. doi:10.1016/j.epsl.2009.10.015.

Waszek, L., Deuss, A., 2011. Distinct layering in the hemispherical seismic velocity structure of Earth's upper inner core. J. Geophys. Res. 116, B12313. doi:10.1029/2011JB008650.

Woodhouse, J.H., Giardini, D., Li, X.D., 1986. Evidence for inner core anisotropy from free oscillations. Geophys. Res. Lett. 13, 1549-1552.

Yoshida, S., Sumita, I., Kumazawa, M., 1996. Growth model of the inner core coupled with the outer core dynamics and the resulting elastic anisotropy. J. Geophys. Res. 101, 28085-28104. doi:10.1029/96JB02700. 

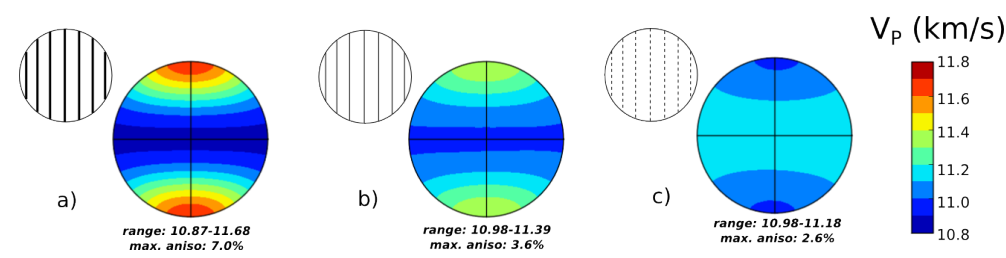

Figure 1: Elastic models used for structural models. a) Elastic moduli of Laio et al. (2000) with a 100\% alignment of the $c$-axes of the polycrystal in the NS directions, later labeled as "strongly" anisotropic. b) Elastic moduli of Laio et al. (2000) with a $50 \%$ alignment of the c-axes of the polycrystal in the NS directions, later labeled as "mildly" anisotropic. c) Elastic moduli of Laio et al. (2000) with an alignment of the c-axes of the polycrystal in the equatorial plane, later labeled as "dentritic". Insets indicate the corresponding simplified representation used in later figures. 

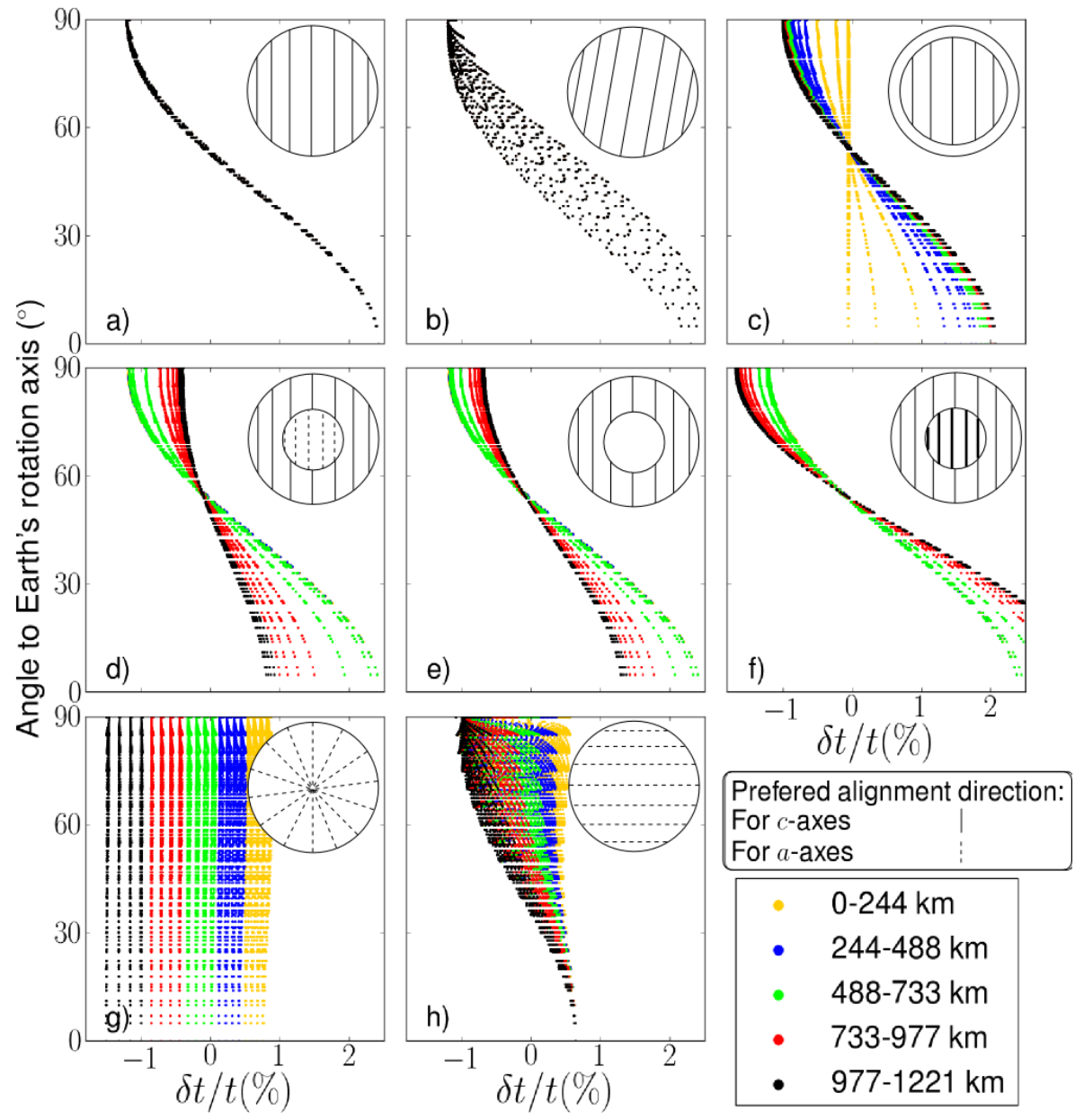

Figure 2: Travel time residuals (in \%) plotted against the angle of the seismic ray to the Earth rotation axis. Results are plotted for different structural inner core models (insets, with lines according to the conventions defined in Fig. 1). Colors indicate the depth of turning point for the ray. 

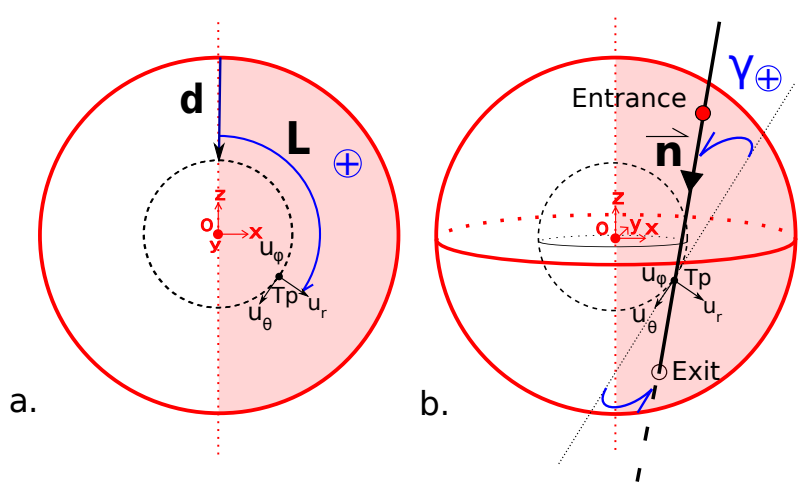

Figure 3: Geometrical construction of inner core seismic rays. They are classified using 3 scalars: the depth of its turning point, $d$, (the deepest point of the ray) $[0-1220 \mathrm{~km}]$, the latitude of its turning point, $L\left[0-180^{\circ}\right]$, and the angle between the ray at its turning point and the meridional plane containing the turning point, $\gamma\left[0-360^{\circ}\right]$. The length of the ray is then divided into straight segments between the entrance and exit points on a 20x72x72 regular grid in $d, L$, and $\gamma$. For each segment, we evaluate the slowness of the material by solving the Christoffel equation with the local elastic tensor. 


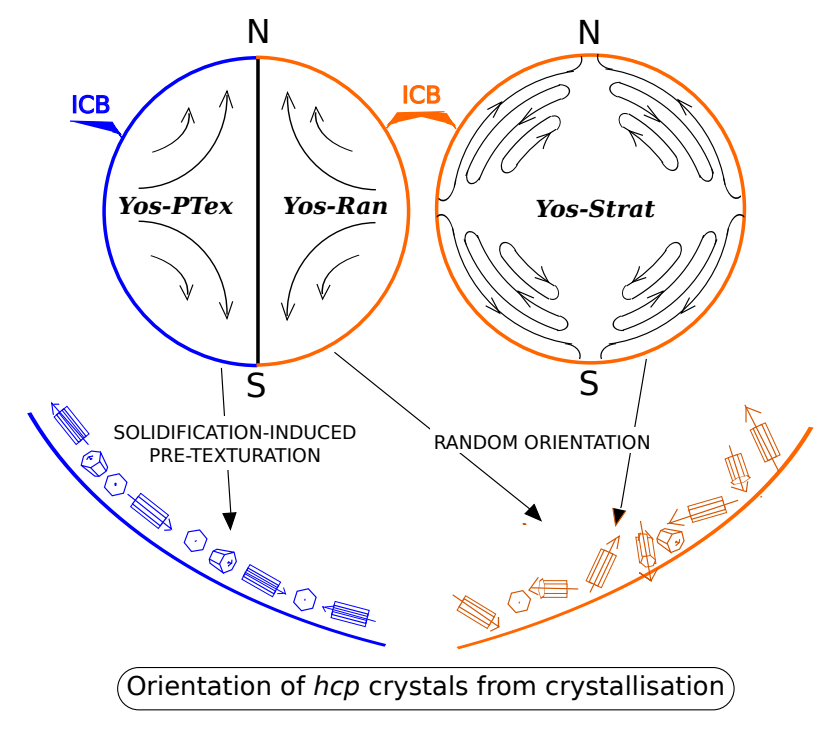

Figure 4: Schematic view of the geodynamical models used in this paper. Yos-Ptex and Yos-Ran are deduced from the quadrupolar model of Yoshida et al. (1996). In Yos-PTex, hcp crystals solidify at the ICB with their $c$-axes lying preferentially in the plane of the ICB (blue) while in Yos-Ran we assume a random solidification texture (red). Yos-Strat is an extension of the model of Yoshida et al. (1996) accounting for a stable density stratification (Deguen and Cardin, 2009). In this case, deformations are significant and solidification textures are quickly erased. The results presented here are therefore limited to random solidification texture. 


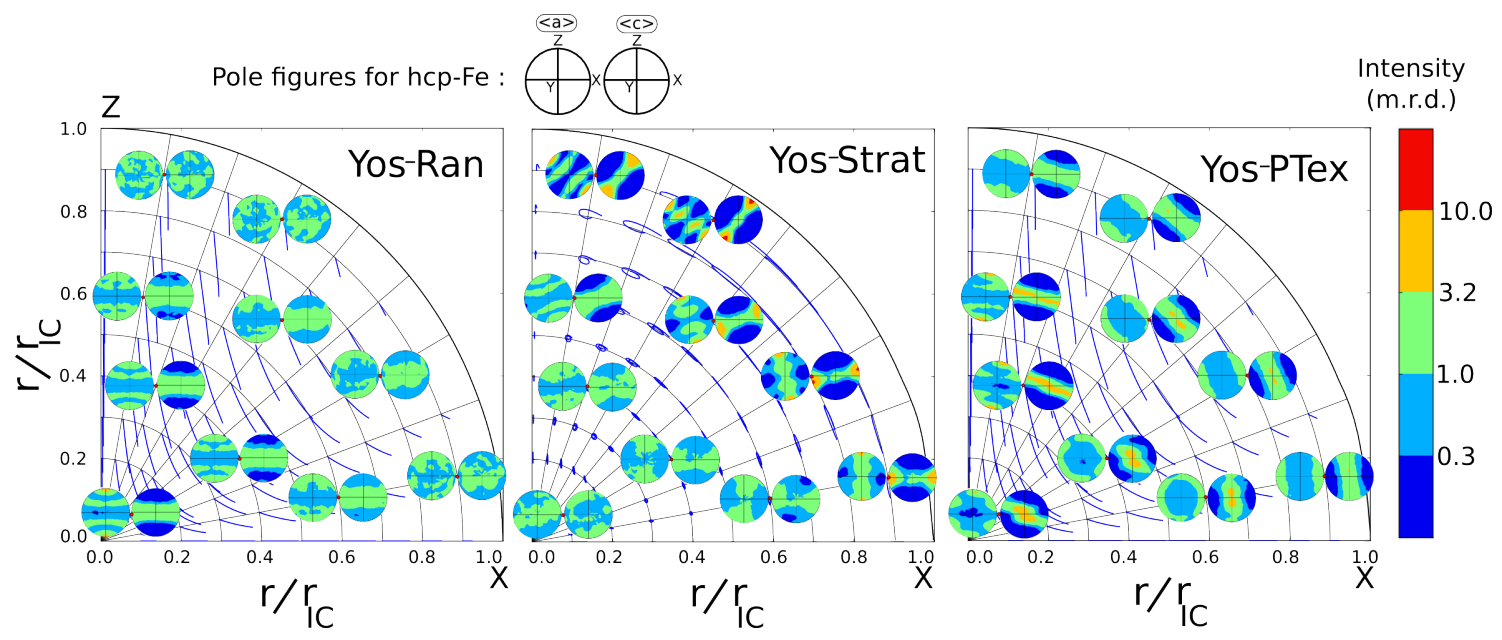

Figure 5: Pole figures of the $a$ and $c$ directions representing present day textures in $\varepsilon$-Fe aggregates at various locations of the inner core. Blue lines are the trajectories of the polycrystalline aggregates after crystallization at the ICB. Contours in multiple of a random distribution (logarithmic scale). a) Yos-Ran, b) Yos-Strat and c) Yos-PTex geodynamical models. 


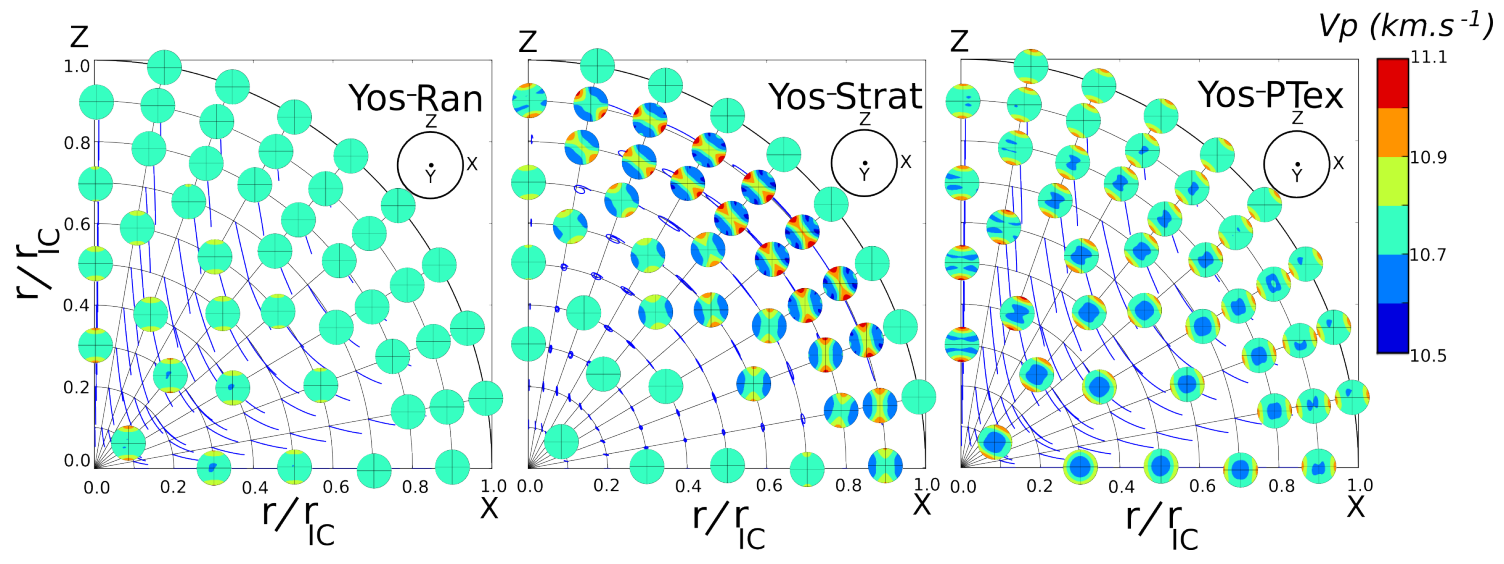

Figure 6: $P$-wave velocity (in $\mathrm{km} / \mathrm{s}$ ) in a meridional plane for geodynamical models Yos-Ran (a), Yos-Strat (b), and Yos-PTex (c). Calculations based on the textures of Fig. 5 and the single crystal elastic moduli of Vočadlo et al. (2009) at $5500 \mathrm{~K}$. 

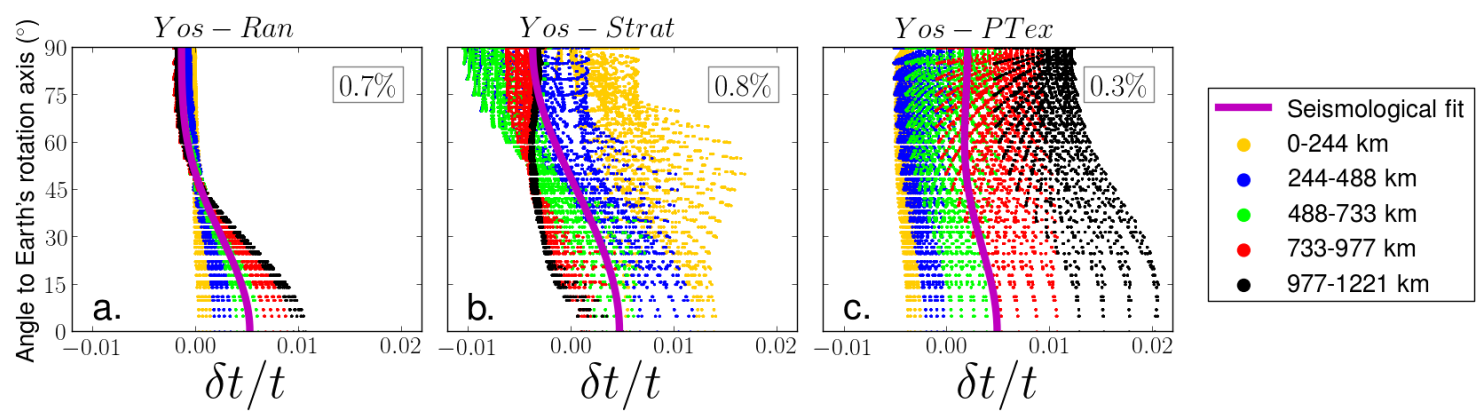

Figure 7: Travel time residuals (dots) plotted against the angle of the seismic ray to the Earth rotation axis for the three geodynamical models of Fig. 4. Colors indicate the depth of turning point of the ray. Thick purple line is the fit of Eq. 2 to the data. Numbers in the insets indicate the average anisotropy for each model. 